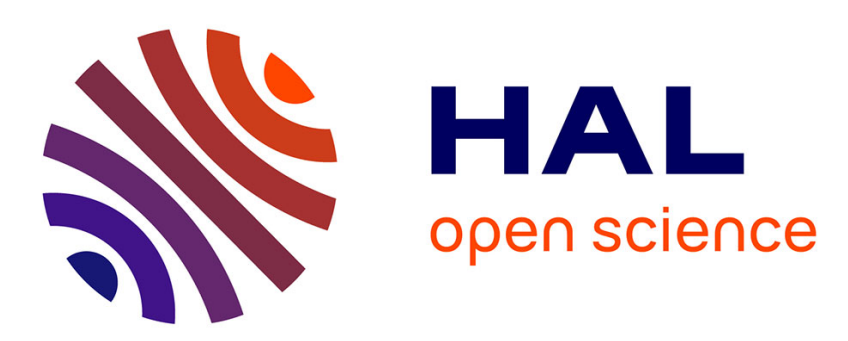

\title{
Investigation of Protective Properties of Organic Layers toward Reactive Oxygen Species
}

\author{
Yara Aceta, Philippe Hapiot, Yann R Leroux
}

\section{To cite this version:}

Yara Aceta, Philippe Hapiot, Yann R Leroux. Investigation of Protective Properties of Organic Layers toward Reactive Oxygen Species. Langmuir, 2019, 35 (49), pp.16210-16216. 10.1021/acs.langmuir.9b02991 . hal-02397309

HAL Id: hal-02397309

https://hal-univ-rennes1.archives-ouvertes.fr/hal-02397309

Submitted on 11 Dec 2019

HAL is a multi-disciplinary open access archive for the deposit and dissemination of scientific research documents, whether they are published or not. The documents may come from teaching and research institutions in France or abroad, or from public or private research centers.
L'archive ouverte pluridisciplinaire HAL, est destinée au dépôt et à la diffusion de documents scientifiques de niveau recherche, publiés ou non, émanant des établissements d'enseignement et de recherche français ou étrangers, des laboratoires publics ou privés. 


\title{
Investigation of Protective Properties of Organic Layers Towards Reactive Oxygen Species (ROS).
}

Yara Aceta, Philippe Hapiot, Yann R. Leroux*

Univ Rennes, CNRS, ISCR - UMR 6226, F-35000 Rennes, France

\begin{abstract}
The antioxidant protective properties of polyaromatic organic layers were evaluated towards Reactive Oxygen Species (ROS) using Scanning Electrochemical Microscopy (SECM) in a foot-printing strategy. The layers were prepared by electrografting of aryldiazonium salts. Where $p$-(methyl)phenyl films show only weak protective properties towards ROS, p-(ethynyl)phenyl films evidence an efficient protection of the covered surfaces. Applied potentials and electrolytes used during ORR are critical parameters to control, to prevent or reduce the influence of ROS production and hence enhancing devices lifetime.
\end{abstract}

KEYWORDS: Reactive Oxygen Species (ROS), Scanning Electrochemical Microscopy (SECM), Aryl Diazonium, Surface Functionalization. 


\section{Introduction}

In a general manner, protective strategies towards Reactive Oxygen Species (ROS) have to be found in order to prevent damages of various devices where ROS could be generated during operation. Biological systems have developed several antioxidant defense strategies to regulate the production of ROS, as superoxide dismutase, ${ }^{1}$ glutathione ${ }^{2}$ or selenoproteins ${ }^{3}$ to name of few. In the literature, many research groups have proposed various protecting strategies towards ROS. As an example, glutathione has been successfully immobilized onto surfaces, ${ }^{4}$ showing high sensitivity towards ROS. ${ }^{5}$ Also, polydopamine nanoparticles (NPs) have been shown to act as a radical scavenger ${ }^{6}$ and have been used to efficiently scavenge ROS in periodontal disease ${ }^{7}$ or during ischemic stroke. ${ }^{8}$ Finally, as a last example, cerium oxide NPs were found also to exhibit antioxidant properties, and have been utilized in biomedical applications ${ }^{9}$ or to enhance polymer exchange membrane (PEM) fuel cells lifetime, where the loss of performance has been especially attributed to the degradation of the membrane by ROS generated during operation. ${ }^{10}$ Even if some of these strategies allowed protecting or enhancing lifetime devices, there is still missing studies about new and cheap protective layers towards ROS. Few years ago, we have found that some organic films deposited on carbon surfaces show irreversible degradations when

exposed to ROS. ${ }^{11,12}$ More recently, Bard and coworkers observed similar degradations of organic films, and used ROS to effectively clean an electrode surface from the adsorption of 4aminothiophenol. ${ }^{13}$ Mirkin et al. also demonstrated that platinum dissolves during ORR in organic and aqueous media, ${ }^{14}$ and that this dissolution generates platinum nanoparticles. ${ }^{15}$ From these observations, in this study, our aim is to investigate the protective properties of organic films towards Reactive Oxygen Species (ROS) generated during Oxygen Reduction Reaction 
(ORR) on platinum. For that purpose, we use Scanning Electrochemical Microscopy (SECM) in a foot-printing strategy.

$\mathrm{SECM}^{16,17}$ has demonstrated all its interest for probing at the local scale a variety of biological, chemical and electrochemical processes occurring at interfaces. ${ }^{18}$ In feedback mode, SECM provides a chemical view of a surface from the solution side and is complementary to other electrochemical methods such as cyclic voltammetry. ${ }^{19}$ Here, the foot-printing strategy is based on the use of SECM in feedback mode when the substrate under investigation is not electrically connected (unbiased conditions). The process involves three main steps: (i) the SECM tip is placed in the close proximity of the organic layer under investigation by registering approach curves with the aid of a redox probe in solution. Because the modified surface is insulating, the tip-substrate distance is derived from the approach curve and compared with the theoretical current-distance curve that only depends on the tip geometry for an insulating surface; (ii) once the SECM tip is in position, ROS are generated at the SECM tip. To do so, the solution with the redox probe is replaced with a clean aqueous electrolytic solution and a potential sufficiently negative to generate ROS (during Oxygen Reaction Reduction, ORR) is applied to the SECM tip ; (iii) finally, to evidence the impact of the ROS on the organic layer, the latter is probed with an indifferent redox mediator either by moving the tip in solution to register an image of the area in the $\mathrm{x}-\mathrm{y}$ plane or by registering approach curves before and after ROS interaction. Different types of approach curves can be obtained (from positive to negative feedback) that sense the ability of the probe to pass through the layer, providing a view of the modifications.

In previous studies, we $\mathrm{e}^{11,12}$ and other research groups ${ }^{20,21,22}$ observed that ROS produced during $\mathrm{O}_{2}$ reduction on a Pt electrode could irreversibly damage various polyphenylene layers for 
patterning purpose. These investigations have also demonstrated the production of extremely reactive radicals in organic and neutral aqueous media during ORR. ${ }^{11}$ Here, we focus on the protective properties of two different organic layers, a $p$-(methyl)phenyl and a $p$-(ethynyl)phenyl film. These two organic layers were selected as they can easily be electro-grafted from their respective aryl diazonium salt parents and form thin and compact organic layers on many substrates. Both organic layers are derivate of hydrocarbons, but their respective chemical nature should induce different reactivity towards oxidation reactions. The study was first performed in basic medium, which avoid the presence of anions other than $\mathrm{OH}^{-}$in the electrolytic solution that are known to react with ROS. ${ }^{23,24,25}$ These experimental conditions allow longer radical lifetime and cleaner test media by limiting the formation of secondary radicals. For comparison, same experiments were also performed in neutral aqueous solution. The role of the different applied potentials is also examined.

\section{Experimental section.}

\subsection{Chemicals and reagents.}

Commercially available reagents were used as received. 4-(ethynyl)benzenediazonium tetrafluoroborate (Eth- $\left.\mathrm{ArN}_{2}{ }^{+} \mathrm{BF} 4\right)^{-26}$ and 4-(methyl)benzenediazonium tetrafluoroborate (Me$\left.\mathrm{ArN}_{2}{ }^{+} \mathrm{BF} 4\right)^{27}$ were synthesized according to published procedures. All aqueous solutions were made employing ultrapure water $(18.2 \mathrm{M} \Omega \mathrm{cm})$.

\subsection{Pyrolytic Photoresist Films (PPFs).}

The fabrication of PPFs consists of a thermal processing of a standard positive photoresist (AZ4562, supplied by Microchemicals), which was previously deposited by spin coating (6000 r.p.m. during 30 s) over a silicon wafer ( $\mathrm{Si}(100)$ ) and heated in an inert atmosphere (95 \% $\mathrm{Ar} / 5$ 
$\% \mathrm{H}_{2}$ ) at rate of $10{ }^{\circ} \mathrm{C} / \mathrm{min}$ up to a temperature of $1100{ }^{\circ} \mathrm{C}$. For complete graphitization of the photoresist material, the annealing was performed during $1 \mathrm{~h} .{ }^{28}$ Finally, the PPFs with typical dimensions of $15 \times 15 \mathrm{~mm}^{2}$ were cleaned in an ultrasonic bath in acetone, methanol and 2propanol, then dried under Argon and stored at room temperature for later use. The resulting PPFs show electrochemical properties close to that observed in glassy carbon electrodes, and low resistivity.

\subsection{Preparation of modified substrates.}

All carbon electrodes were modified in a $10^{-2} \mathrm{~mol} \mathrm{~L}^{-1}$ acetonitrile (MeCN) solution of the corresponding aryldiazonium salt (Eth- $\mathrm{ArN}_{2}{ }^{+} \mathrm{BF}^{-}$or $\mathrm{Me}-\mathrm{ArN}_{2}{ }^{+} \mathrm{BF} 4^{-}$) by fixed potential chronoamperometry during 300 seconds at $-0.8 \mathrm{~V}$ vs SCE. The solution was previously deaerate with Argon (20 min). The modified electrodes were stirred 20 min in MeCN solution, rinsed with acetone and dried under argon. Preliminary experiments were performed on glassy carbon electrodes. Prior to modification, they were polished successively with 2400 and 4000 silicon carbide abrasive discs (Struers) and ultrapure water and thoroughly rinsed with ultrapure water and acetone. For modifying PPFs, no pre-polishing stage is necessary.

\subsection{Electrochemical instrumentation.}

All surface modifications were performed using a standard three-electrode setup at a controlled potential with a potentiostat Autolab PGSTAT 302N (Metrohm). The set of electrodes consisted of glassy carbon (GC) disk electrodes (IJ Cambria, surface area $0.03 \mathrm{~cm}^{2}$ ) or small squares $\left(15 \times 15 \mathrm{~mm}^{2}\right)$ of PPFs as working electrodes, a platinum mesh as the auxiliary electrode, and a saturated calomel electrode (SCE, $\mathrm{KCl}$ sat.) as reference. The latter was isolated from the working solution employing a salt bridge, which contained the electrolyte solution. When 
necessary, a conventional gas inlet was used for bubbling argon inside the electrochemical cell to displace oxygen.

SECM experiments were performed with a three-electrode setup, comprising a $10 \mu \mathrm{m}$ diameter Pt UME (IJ Cambria) with a RG = 7, a Pt wire as the auxiliary electrode, and a homemade $\mathrm{Ag} / \mathrm{AgCl}\left(0.1 \mathrm{~mol} \mathrm{~L}^{-1} \mathrm{KCl}\right)$ electrode as reference. Measurements were performed using a homemade setup similar to that described in reference [29]. The SECM setup is equipped with an adjustable stage for the tilt angle correction and is controlled by the SECMx software written by G. Wittstock et al. ${ }^{30}$ For SECM imaging, the tip was positioned at a fix distance of 5 $\mu \mathrm{m}$, recording approach curve in the top left corner. SECM images and approach curves were recorded using a CHI910 potentiostat from $\mathrm{CH}$ Instruments.

\subsection{Atomic Force Microscopy.}

Atomic force microscopy (AFM) experiments were performed on modified PPF substrates using a NT-MDT Ntegra microscope. Topographic images were obtained in semi-contact mode using ACTA tips (AppNano Inc.) for which the resonance frequency is around $300 \mathrm{kHz}$. The images were treated using Gwyddion open-source software. ${ }^{31}$ The roughness of the organic films deposited on PPF substrates was estimated, according to the standard ISO 4287/1-1997, as the average of the measured height deviations taken within the image (Root Mean Square (RMS) roughness).

\subsection{Spectroscopy Ellipsometry.}

The thickness of the organic films was measured by Spectroscopy Ellipsometry with an SE ellipsometer (J.A. Woollam, Co.) using a model-based analysis approach. Prior to modification with the aryldiazonium molecule, the SE data of the bare substrate were registered to build a substrate optical model which represents the nominal structure of the non-modified material; the 
refractive index (n) and the extinction coefficient (k) were 2.4 and 1.1 for bare PPF electrodes. This substrate model was used after modification to establish the contribution of the substrate to the SE experimental data and thus, estimate the thickness of the organic layer according to the Cauchy model. Finally, the goodness of the fit was quantified by the Mean Squared Error (MSE) to validate our results. In all cases, the fitting reproduced the structures and features of the SE experimental data. The values of thicknesses obtained are the average of ten measurements performed on three different PPF substrates. 


\section{Results and Discussion.}

\subsection{Characterization of the organic layers.}

We considered two different poly-aromatic films that were electrodeposited on carbon substrates (pyrolized photoresist films, PPF). They differ by the presence of an unsaturated bond: a $p$-(methyl)phenyl and a p-(ethynyl)phenyl films. In both cases, a polyaromatic structure is obtained as confirmed by our analysis and according to the literature ${ }^{32,33}$. In the case of the $p$ (ethynyl)phenyl multilayer films, the structure integrates acetylene groups. This feature is expected to confer a different sensitivity of this film towards ROS in solution. The $p$ (methyl)phenyl-based structure could be also described as a polyaromatic multilayer, but in this case, it integrates methyl groups. It is known that methyl terminal groups offer few opportunities for organic chemistry compared to acetylene terminal groups, ${ }^{34}$ then a different behavior could be expected in the presence of oxidants.

a)

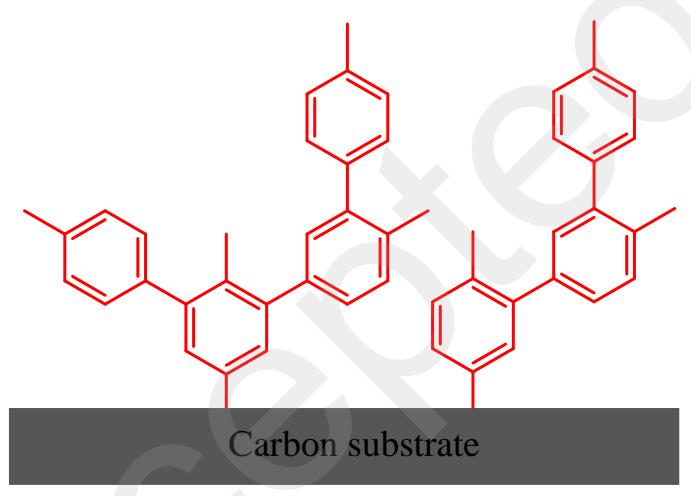

b)

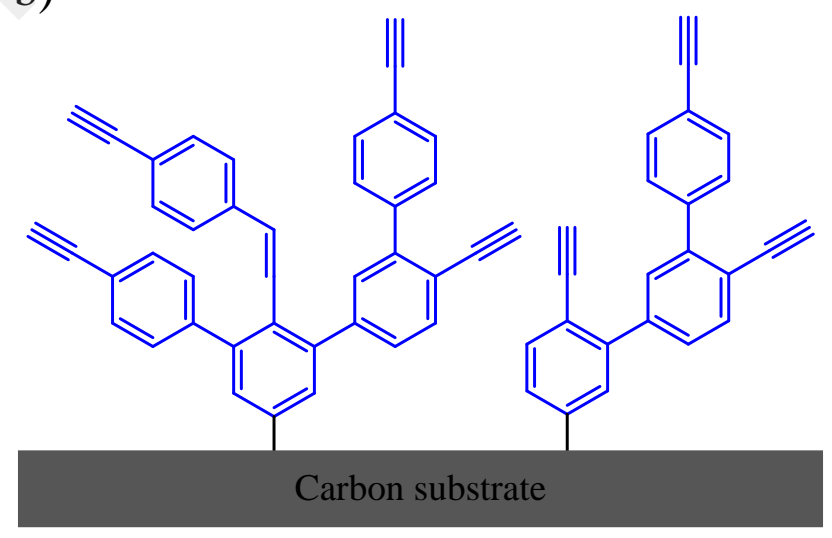

Scheme 1. Schematic representation of the structure of the organic films deposited on carbon substrates by the electroreduction of (a) p-(methyl)phenyl diazonium salts and (b) $p$ (ethynyl)phenyl diazonium salts. 
Both organic films are easily prepared on PPF surfaces by electro-reduction of the corresponding aryldiazonium cations in acetonitrile $(\mathrm{MeCN})+0.1 \mathrm{~mol} \mathrm{\textrm {L } ^ { - 1 }} n$ tetrabutylammonium hexafluorophosphate $\left(n \mathrm{Bu}_{4} \mathrm{NPF}_{6}\right)$ solutions under self-inhibition conditions (long time electrolysis and large concentration of precursor, see Experimental section for details). In these conditions, thick organic layers with strong blocking properties towards electron transfer of redox probes in solution are obtained. The formation of multi-layers by reduction of these compounds has been reported in the literature but the quality of the film is highly dependent on the experimental conditions. ${ }^{35,36}$ To determine the characteristics of these films, we examined their blocking properties by cyclic voltammetry, and their homogeneity, roughness and thickness by Atomic Force Microscopy (AFM) and spectroscopic ellipsometry (SE).
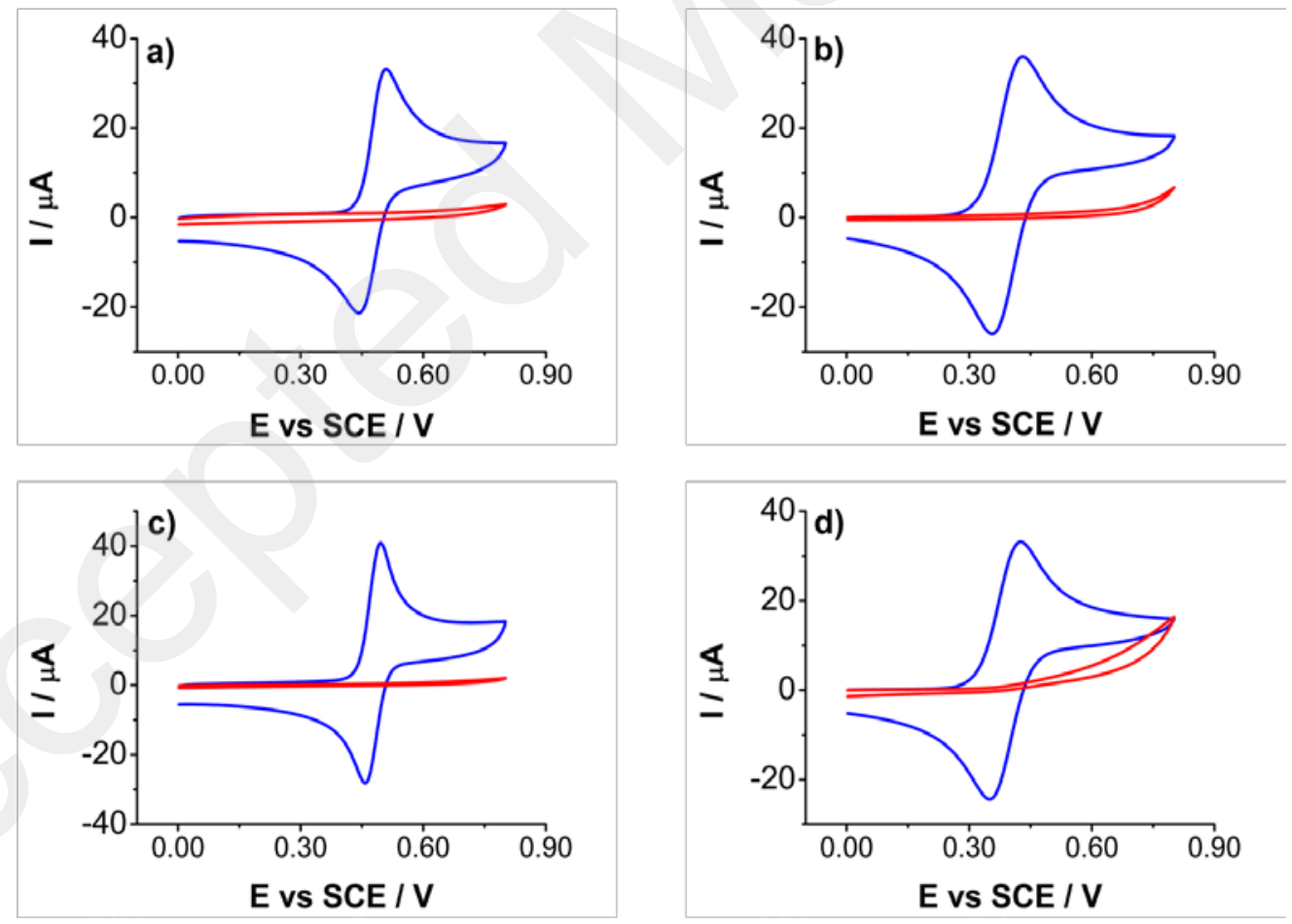

Figure 1. Cyclic voltammograms of a glassy carbon electrode in an (a, c) aqueous solution of $10^{-}$ ${ }^{3}$ mol L ${ }^{-1}$ dopamine $+0.1 \mathrm{~mol} \mathrm{~L}^{-1} \mathrm{H}_{2} \mathrm{SO}_{4}$ and (b, d) an acetonitrile solution of $10^{-3} \mathrm{~mol} \mathrm{~L}^{-1}$ 
ferrocene $+0.1 \mathrm{~mol} \mathrm{~L}^{-1} n \mathrm{Bu}_{4} \mathrm{NPF}_{6}$ before (blue line) and after (red line) electro-grafting by chronoamperometry (at - $0.8 \quad \mathrm{~V}$ vs SCE during 300 seconds) with (a, b) 4(ethynyl)benzenediazonium tetrafluoroborate and (c, d) 4-(methyl)benzenediazonium tetrafluoroborate. Scan rate $0.1 \mathrm{~V} \mathrm{~s}^{-1}$.

Dopamine and ferrocene were used as redox probes in solution to investigate the blocking properties of the modified surfaces. Dopamine undergoes a reversible oxidation to o-quinone in acidic medium but it needs to be absorbed onto the electrode surface for rapidly transferring its charge, which makes dopamine a very sensitive and suitable redox species for probing the quality of organic films (presence of pinholes or defects), by discriminating between direct and indirect charge transfer. ${ }^{37,38}$ Ferrocene also undergoes a reversible oxidation but contrarily to dopamine, it could transfer its charge by tunneling through the organic layer, meaning that ferrocene does not require to be in contact with the electrode substrates for transferring its charge. ${ }^{37}$ As shown in Figure 1, after modification of the PPF surfaces with $p$-(methyl)phenyl and p-(ethynyl)phenyl diazonium salts, both ferrocene and dopamine oxidation are totally inhibited on the modified PPF surfaces. This indicates that both organic films are multilayers, free of defects or pinholes, and that they are thick enough to inhibit the electron transfer by tunneling of ferrocene. In connection with the observed electrochemical behavior, the formation of defect-free covering was confirmed by AFM imaging on both modified PPF surfaces (Figure 2). The topography and surface roughness increase after the grafting associated to the disordered and dendritic growth of these organic films. Analyses of thickness by ellipsometry yielded similar values for both $p$-(methyl)phenyl and p-(ethynyl)phenyl films of $5.6 \mathrm{~nm}$ and $6.8 \mathrm{~nm}$ 
respectively (Table 1). Therefore, both layers present similar surface features and blocking properties towards the electron transfer of redox probes in solution.

a)

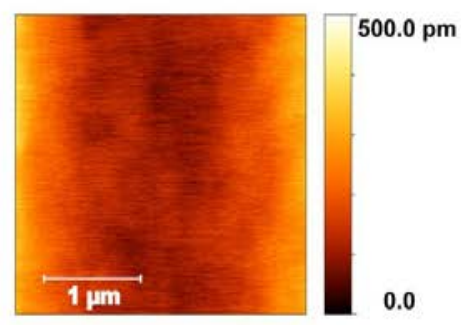

b)

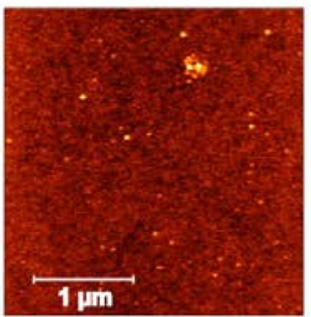

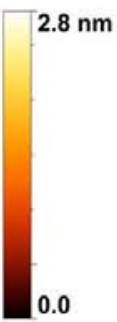

c)

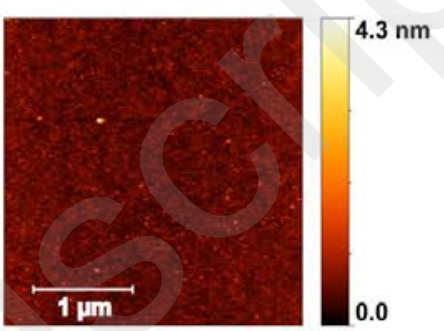

Figure 2. AFM topographic images of (a) bare PPF, (b) p-(methyl)phenyl-modified PPF and (c) p-(ethynyl)phenyl-modified PPF.

Table 1. Roughness and thickness values of the $p$-(methyl)phenyl-modified PPF (Me-Ar-PPF) and $p$-(ethynyl)phenyl-modified PPF (Eth-Ar-PPF) and the bare PPF for comparison. Values are the average of three different measurements performed on three different samples.

\begin{tabular}{|l|l|l|l|}
\hline Surface properties & Bare PPF & Me-Ar-PPF & Eth-Ar-PPF \\
\hline Roughness - AFM / pm & $61 \pm 3$ & $132 \pm 8$ & $154 \pm 10$ \\
\hline Thickness - SE / nm & - & $5.6 \pm 0.7$ & $6.8 \pm 0.3$ \\
\hline
\end{tabular}

\subsection{Protective properties of organic layers.}

The protective properties of the organic films were investigated in two different aqueous electrolyte solutions: $10^{-2} \mathrm{~mol} \mathrm{~L}^{-1} \mathrm{KOH}$ and $10^{-2} \mathrm{~mol} \mathrm{~L}^{-1} \mathrm{KPF}_{6}$ aqueous solutions for comparison. 
We used low concentrations of electrolyte to extend the lifetime of radicals in solution that could react with the electrolyte but also with the impurities present in the salts to form secondary radicals. ORR was performed with the concentration of $\mathrm{O}_{2}$ naturally dissolved in these media under air. The experimental concentration of oxygen was derived from the diffusion-limited current at the platinum ultramicroelectrode (UME) during the reduction of $\mathrm{O}_{2}$ by cyclic voltammetry in these media (Figure 3). The steady-state limit current at a disk ultramicroelectrode is related to the concentration of the electroactive species by the equation: $\mathrm{i}_{\mathrm{lim}}=4 \mathrm{nFDaC}$ where $\mathrm{a}$ is the radius of the platinum UME (5 $\mu \mathrm{m}$ radius), $\mathrm{C}$ is the concentration of oxygen and $\mathrm{D}$ is the diffusion coefficient of $\mathrm{O}_{2}\left(1.97 \times 10^{-5} \mathrm{~cm}^{2} \mathrm{~s}^{-1}\right.$ from the literature $) .{ }^{39,40}$ It was estimated a concentration of $\mathrm{O}_{2}$ of $8.50 \times 10^{-4} \mathrm{~mol} \mathrm{~L}^{-1}$ in $10^{-2} \mathrm{~mol} \mathrm{~L}^{-1} \mathrm{KOH}$ and $8.92 \times 10^{-4} \mathrm{~mol}$ $\mathrm{L}^{-1}$ in $10^{-2} \mathrm{~mol} \mathrm{~L}^{-1} \mathrm{KPF}_{6}$. Solubility of $\mathrm{O}_{2}$ in aqueous electrolyte solutions range between $10^{-3}$ and $10^{-6}$ mol L ${ }^{-1}$, depending on the partial pressure of $\mathrm{O}_{2}$ and the concentration of electrolyte at 298 $\mathrm{K}^{41,42}$
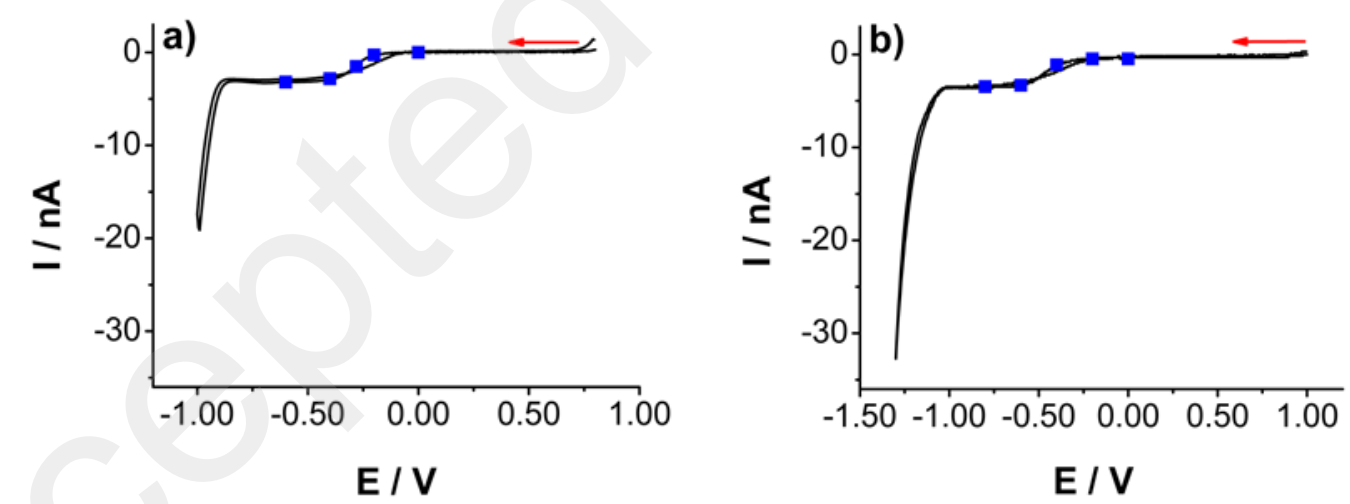

Figure 3. Steady-state cyclic voltammetry of $\mathrm{O}_{2}$ in (a) $10^{-2} \mathrm{~mol} \mathrm{~L}^{-1} \mathrm{KOH}$ and (b) $10^{-2} \mathrm{~mol} \mathrm{~L}^{-1}$ $\mathrm{KPF}_{6}$ aqueous solution on a platinum ultramicroelectrode (5 $\mu \mathrm{m}$ radius). Blue solid squares mark the sequence of applied potentials studied by SECM. Potentials are referred to Ag/AgCl pseudoreference electrode. Scan rate $0.02 \mathrm{~V} \mathrm{~s}^{-1}$. 
Figures 4a-d show the SECM experiments performed in $10^{-2} \mathrm{~mol} \mathrm{~L}^{-1} \mathrm{KOH}$, before (Figures 4 a, b) and after ORR (Figures 4c,d). Figures 4e-h show the SECM experiments performed in $10^{-2}$ mol L ${ }^{-1} \mathrm{KPF}_{6}$, before (Figures 4e,f) and after ORR (Figures 4g,h). SECM images on the left side show the experiments performed on the p-(ethynyl)phenyl film (Figures 4a,c,e,g); SECM images on the right side, the ones performed on the $p$-(methyl)phenyl film (Figures $4 \mathrm{~b}, \mathrm{~d}, \mathrm{f}, \mathrm{h}$ ). In each media, we studied the effect of the applied potential during the ORR performed at the Pt tip electrode that is a polycrystalline surface. The half-wave potential of oxygen reduction in the basic solution was - $0.28 \mathrm{~V}$ vs $\mathrm{Ag} / \mathrm{AgCl}$, and - $0.44 \mathrm{~V}$ vs $\mathrm{Ag} / \mathrm{AgCl}$ in the neutral solution $\left(10^{-2}\right.$ mol L-1 $\mathrm{KPF}_{6}$ ). We used a transient method for the production of the ROS. Each applied potential to the tip electrode was composed of a sequence of two pulses in chronoamperometry, for a total duration of 20 seconds, all started from $0 \mathrm{~V}$ to the applied potential. Four different applied potentials ranging from $0 \mathrm{~V}$ (before ORR, not shown) to the plateau of the ORR were considered. All potentials studied by SECM in each media are marked in the voltammograms of Figure 3 as blue solid squares. 

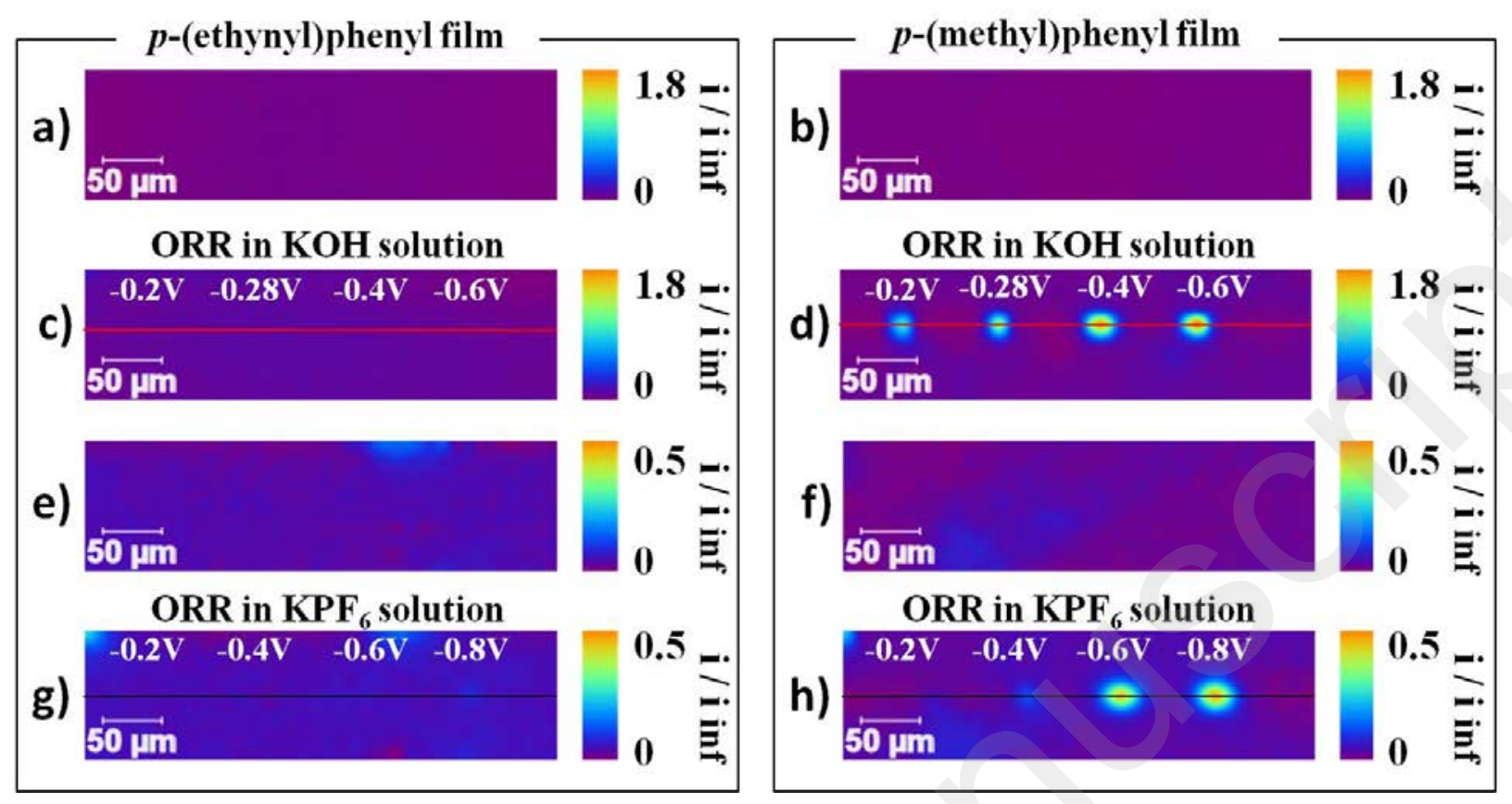

Figure 4. SECM images (400 x $\left.200 \mu \mathrm{m}^{2}\right)$ registered with a Pt UME (5 $\mu \mathrm{m}$-radius) in $10^{-3} \mathrm{~mol} \mathrm{~L}^{-}$ ${ }^{1} \mathrm{Fc}(\mathrm{MeOH})_{2}$ as redox mediator in $0.1 \mathrm{~mol} \mathrm{~L}^{-1} \mathrm{H}_{2} \mathrm{SO}_{4}$ before (a, b, e, f) and after (c, d, g, h) performing ORR in the vicinity $(5 \mu \mathrm{m})$ of the $p$-(ethynyl)phenyl film (a, c, e, g) and $p$ (methyl)phenyl film (b, d, f, h). Various fixed reductive potentials were applied during ORR, from $0.0 \mathrm{~V}$ to $-0.6 \mathrm{~V}$ vs $\mathrm{Ag} / \mathrm{AgCl}$ in $10^{-2} \mathrm{~mol} \mathrm{~L}^{-1} \mathrm{KOH}$ solution (c, d) and from $0.0 \mathrm{~V}$ to $-0.8 \mathrm{~V}$ vs Ag/AgCl in $10^{-2} \mathrm{~mol} \mathrm{~L}^{-1} \mathrm{KPF}_{6}$ solution (g, h). Y-axes display the normalized tip current (i/i $\mathrm{i}_{\text {inf }}$ ). Note that the background current was subtracted for comparison, where the background current corresponds to the value of normalized tip current when the UME tip is at $5 \mu \mathrm{m}$ to the organic film.

As shown in Figure 4, before ORR, all organic films show a totally insulating behavior toward electron transfer when $\mathrm{Fc}(\mathrm{MeOH})_{2}$ is used as probe, displaying a fairly homogeneous electrochemical response on the surface of the samples. No surface defects or large current variations were evidenced in both neutral and basic solution. Dopamine was not used in these experiments as it is too sensitive to oxygenated species and can lead to a misinterpretation of the 
results. After exposition to ROS, no modification was observed on the p-(ethynyl)phenyl film for all applied reductive potentials; the layer remains totally insulating and homogenous, still blocking the electron transfer of $\mathrm{Fc}(\mathrm{MeOH})_{2}$ in solution. This behavior is observed in both neutral and basic media. On the contrary, on p-(methyl)phenyl films, areas where the electron transfer rate of $\mathrm{Fc}(\mathrm{MeOH})_{2}$ increases are clearly visible after exposure to ROS, for all applied reductive potentials in $\mathrm{KOH}$ solution and for the two most reductive potentials in $\mathrm{KPF}_{6}$. This increase in electron transfer rate reflects a change in the topology of the film where the areas under the SECM tip are now sufficiently thin to allow electron transfer from $\mathrm{Fc}(\mathrm{MeOH})_{2}$ cation in solution to the underneath carbon substrate by electron tunneling, or direct transfer. This evidences the degradation of the organic layer by the ROS electro-generated at the SECM tip. For a more quantitative comparison between experiments, it is interesting to examine the currentdistance profiles. Figure 5 shows the normalized current $\left(\mathrm{i} / \mathrm{i}_{\mathrm{inf}}\right)$ versus the distance covered by the SECM tip on the $\mathrm{x}$-axis $(400 \mu \mathrm{m})$ in the two aqueous electrolyte solutions after ORR. As previously indicated, no modification of the $p$-(ethynyl)phenyl film was observed under any of the experimental conditions studied whatever the applied potentials and electrolyte solutions (Figure 5a). On contrast, the $p$-(methyl)phenyl film shows a different behavior towards ROS in solution, and this behavior varies with both the applied potential and the electrolyte solution. As seen on Figure 5b, the extent of the modification is much more important in alkaline medium (red) than in neutral solution (black). The normalized current associated to the electron transfer of $\mathrm{Fc}(\mathrm{MeOH})_{2}$ is more than three time larger when the applied potentials are in the plateau of the ORR. 

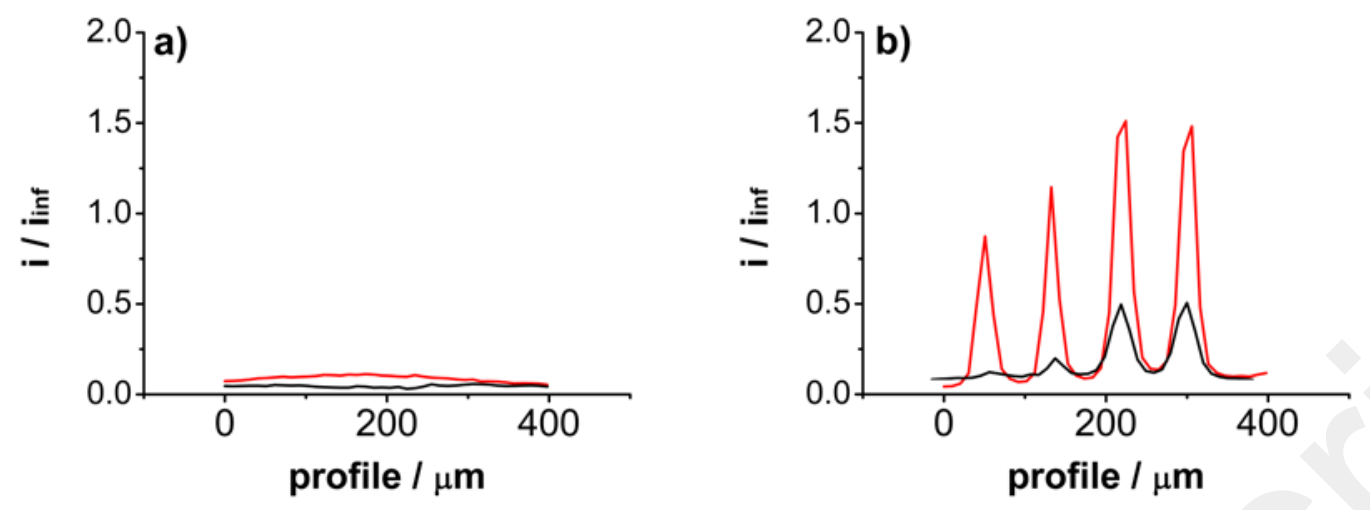

Figure 5. Current-distance profiles of (a) a p-(ethynyl)phenyl film and (b) a $p$-(methyl)phenyl film deposited onto PPF substrates after ORR in $10^{-2}$ mol L ${ }^{-1} \mathrm{KOH}$ (red) and $10^{-2} \mathrm{~mol} \mathrm{~L}^{-1} \mathrm{KPF}_{6}$ (black) solutions. Profiles drawn from Figures 4 c, d, g and h. Data analysis was performed with Gwyddion software. ${ }^{31}$ Note that the background current was subtracted for comparison, where the background current corresponds to the value of normalized tip current when the UME tip is at $5 \mu \mathrm{m}$ to the organic film.

\subsection{Discussion.}

The $p$-(ethynyl)phenyl film does not undergo modification after exposition to ROS, while $p$ (methyl)phenyl film is considerably affected. Applying a more cathodic potential to the platinum UME, clearly enhanced the damage on the organic film since the rate of generation of ROS increases. Notice that the size of the damages remains unchanged and in the order of the diffusion cone of the SECM tip while the contrast (normalized tip current) of the damages increase when applying more cathodic potential. This is in agreement with the behavior expected in a steady state diffusing system. ${ }^{43}$ It is first noticeable that the small difference in layer thickness between the two films cannot explain the extent of the modification observed. The $p$ (ethynyl)phenyl film is $20 \%$ thicker than the $p$-(methyl)phenyl one, while degradations of the 
latter is largely enhanced (normalized tip current increases by at least $300 \%$ ) when ORR is performed in the plateau of oxygen reduction (see Figure 4 and 5). The different behaviors between the organic films could be ascribed to changes of reactivity of the films towards radicals produced during ORR on Pt. As demonstrated in our earlier studies, superoxyde $\left(\mathrm{O}_{2}{ }^{\circ}\right)^{5}$ is not responsible for the degradation of any organic films. Furthermore, the effects of hydrogen peroxide $\left(\mathrm{H}_{2} \mathrm{O}_{2}\right)$ on both organic layers were evaluated (see supporting information) and any degradation was observed. From here, we concluded that the most likely responsible for the degradation of the organic films is the radical $\mathrm{HO}^{\circ}$, being one of the most reactive radical among all the ROS formed during ORR at the platinum electrode. ${ }^{11}$ Reactions between $\mathrm{OH}^{*}$ and aromatic molecules have been extensively studied by pulse radiolysis ${ }^{44,45,46}$ including molecules which incorporate terminal acetylene and methyl functions. Three possible reactions between $\mathrm{HO}^{\circ}$ and organic molecules were generally identified: (i) the abstraction of $\mathrm{H}$ atoms, (ii) the electrophilic addition on unsaturated bonds, and (iii) the electron transfer process. ${ }^{47}$ By analogy, it is expected that the reaction between the $\mathrm{HO}^{\circ}$ and the acetylene groups in the $p$ (ethynyl)phenyl film mainly proceeds by electrophilic addition to the carbon-carbon triple bond leading to the corresponding enol form of the formylmethyl radical that is stabilized by resonance, ${ }^{48,49}$ while hydrogen abstraction is the dominant reaction pathway for the attack of $\mathrm{HO}^{\circ}$ on the terminal methyl function in the $p$-(methyl)phenyl film. ${ }^{50,51}$ In this case, the abstraction of $\mathrm{H}$ atoms results in the formation of benzylic methylene radicals, which are species much more reactive than the stabilized intermediate in the $p$-(ethynyl)phenyl film. These radicals could therefore induce other radical reactions (propagation reactions) through the organic film, thus contributing to the further oxidative degradation of the $p$-(methyl)phenyl film and leading to the different reactivity observed for the two films. Note that the conjugated polyaromatic 
structure of both organic films contributes to the stabilization of both benzylic methylene and phenyl acetylene radicals. Concerning the polyaromatic structure, hydroxyl radicals are strong electrophilic species that can also directly attack the aromatic ring to produce phenolic derivatives. In this case, the inductive effect and the $\pi$ - donor and acceptor character of the substituent in the aromatic ring should be considered. The greater donor character of the methyl group favors to a larger extent the electrophilic attack of the $\mathrm{HO}^{*}$ to the aromatic ring, reinforcing the evidences of a faster degradation of the $p$-(methyl)phenyl film by comparison with the $p$ (ethynyl)phenyl film. Hence, the unsaturated bonds integrated in the p-(ethynyl)phenyl films seem to prevent or delay the oxidative damage of the polyaromatic structure during the exposition to ROS; while $p$-(methyl)phenyl films is more easily damaged by species generated during the ORR on platinum.

Furthermore, there are some noticeable differences between the reactions performed in the two different electrolytes. The degradation of $p$-(methyl)phenyl films is largely enhanced (normalized tip current increases by at least $300 \%$ ) in alkaline solution by comparison with the same electrochemical procedure when performed in the neutral solution $\left(\mathrm{KPF}_{6}\right)$. This can be explained in terms of the mechanism of the oxygen reduction reaction in the different media studied. In alkaline solution, a 1-electron mechanism from $\mathrm{O}_{2}$ to $\mathrm{O}_{2}{ }^{--}$has been proposed. In this medium, superoxide exhibits a significant lifetime since high concentration of $\mathrm{OH}^{-}$promote the existence of superoxide according with the Equation 1, while the lifetime of superoxide in presence of protons is too short and should disappears by protonation to form $\mathrm{HO}_{2}{ }^{\circ}$ that in turn undergoes disproportionation to $\mathrm{H}_{2} \mathrm{O}_{2} .{ }^{52}$

$$
2 \mathrm{O}_{2}^{--}+2 \mathrm{H}_{2} \mathrm{O} \rightleftharpoons \mathrm{H}_{2} \mathrm{O}_{2}+\mathrm{O}_{2}+2 \mathrm{OH}^{-}
$$


As consequence, an almost complete conversion to $\mathrm{H}_{2} \mathrm{O}_{2}$ occurs in the $\mathrm{KPF}_{6}$ aqueous solution which explains the lowest reactivity observed in this medium; as we have already demonstrated, $\mathrm{H}_{2} \mathrm{O}_{2}$ is not able to modify the organic layer and the potentials applied during the experiment are not enough reductive to reduce $\mathrm{H}_{2} \mathrm{O}_{2}$ to $\mathrm{OH}^{*}$. On the contrary, a longer lifetime of the superoxide radical in an alkaline medium (less favorable hydrogen peroxide formation) would allow the production of $\mathrm{OH}^{*}$ or other secondary radicals by interaction with the solvent, explaining the highest extent of the phenomena in alkaline media and the different reactivity between alkaline and neutral media. Also, as quoted in the introduction, similar anions than $\mathrm{PF}_{6}{ }^{-}$in solution are known to react strongly with ROS. ${ }^{23,24,25}$ In our conditions, these reactions could lead to an important decrease of the ROS lifetime in the medium. These secondary reactions compete with the reaction of the ROS with the deposited organic layer leading to an apparent enhancement of the protective properties in this media. 


\section{Conclusion.}

The antioxidant properties of two different organic films, a $p$-(ethynyl)phenyl and a $p$ (methyl)phenyl films, were investigated towards ROS in basic and neutral media. Non-intuitive differences in their reactivity were observed. p-(Ethynyl)phenyl film does not undergo modification after exposure to ROS, while $p$-(methyl)phenyl film is considerably affected and in different media. These different behaviors could be attributed to a "radical scavenging" property of the unsaturated bonds present in the p-(ethynyl)phenyl film. It seems to prevent or delay the oxidative damages of the polyaromatic structure during the exposition to ROS, unlike that observed in the $p$-(methyl)phenyl film. It will be interesting in further investigations to probe other polymer systems bearing unsaturated bonds, checking if they present or not similar effect. These findings open the way to new protection layers and materials towards ROS.

\section{ASSOCIATED CONTENT}

Supporting Information. SECM methodology is described in detail. Control experiments were performed to rule out possible damage by $\mathrm{H}_{2} \mathrm{O}_{2}$ and a possible Fenton-like reaction on both organic films. The following file is available free of charge.

\section{AUTHOR INFORMATION}

\section{Corresponding Author}

*yann.leroux@univ-rennes1.fr

\section{Author Contributions}


The manuscript was written through contributions of all authors. All authors have given approval to the final version of the manuscript. All authors contributed equally.

\section{ACKNOWLEDGMENT}

Y.A. thanks the French Ministry of Research for financial support.

\section{REFERENCES}

[1] Grene Alscher, R.; Erturk, N.; Heath, L. S. Role of superoxide dismutases (SODs) in controlling oxidative stress in plants. J. Exp. Bot. 2002, 53, 1331-1341.

[2] Espinosa-Diez, C.; Miguel, V.; Mennerich, D.; Kietzmann, T.; Sánchez-Pérez, P.; Cadenas,

S.; Lamas, S. Antioxidant responses and cellular adjustments to oxidative stress. Redox Biol. 2015, 6, 183-197.

[3] Steinbrenner, H.; Sies, H. Protection against reactive oxygen species by selenoproteins. Biochim. Biophys. Acta 2009, 1790, 1478-1485.

[4] Griveau, S.; Mercier, D.; Vautrin-Ul, C.; Chausse, A. Electrochemical grafting by reduction of 4-aminoethylbenzenediazonium salt: Application to the immobilization of (bio)molecules. Electrochem. Commun. 2007, 9, 2768-2773

[5] Latus, A.; Noel, J.-M.; Volanschi, E.; Lagrost, C.; Hapiot, P. Scanning Electrochemical Microscopy Studies of Glutathione-Modified Surfaces. An Erasable and Sensitive-to-Reactive Oxygen Species Surface. Langmuir 2011, 27, 11206-11211. 
[6] Ju, K.-Y.; Lee, Y.; Lee, S.; Park, S. B.; Lee, J.-K. Bioinspired Polymerization of Dopamine to Generate Melanin-Like Nanoparticles Having an Excellent Free-Radical-Scavenging Property. Biomacromolecules 2011, 12, 625-632.

[7] Bao, X.; Zhao, J.; Sun, J.; Hu, M.; Yang, X. Polydopamine Nanoparticles as Efficient

Scavengers for Reactive Oxygen Species in Periodontal Disease. ACS Nano 2018, 12, 88828892.

[8] Liu, Y.; Ai, K.; Ji, X.; Askhatova, D.; Du, R.; Lu, L.; Shi, J. Comprehensive Insights into the Multi-Antioxidative Mechanisms of Melanin Nanoparticles and Their Application to Protect Brain from Injury in Ischemic Stroke. J. Am. Chem. Soc. 2017, 139, 856-862.

[9] Dhall, A.; Self, W. Cerium Oxide Nanoparticles: A Brief Review of Their Synthesis Methods and Biomedical Applications. Antioxidants 2018, 7, 97.

[10] Rodgers, M. P.; Bonville, L. J.; Kunz, H. R.; Slattery, D. K.; Fenton, J. M. Fuel Cell Perfluorinated Sulfonic Acid Membrane Degradation Correlating Accelerated Stress Testing and Lifetime. Chem. Rev. 2012, 112, 6075-6103.

[11] Noèl, J.-M.; Latus, A.; Lagrost, C.; Volanschi, E.; Hapiot, P. Evidence for OH Radical Production during Electrocatalysis of Oxygen Reduction on Pt Surfaces: Consequences and Application. J. Am. Chem. Soc. 2012, 134, 2835-2841.

[12] Lhenry, S.; Leroux, Y. R.; Hapiot, P. Use of Catechol As Selective Redox Mediator in Scanning Electrochemical Microscopy Investigations. Anal. Chem. 2012, 84, 7518-7524. 
[13] Percival, S. J.; Dick, J. E.; Bard, A. J. Cathodically dissolved platinum resulting from the $\mathrm{O} 2$ and $\mathrm{H} 2 \mathrm{O} 2$ reduction reactions on platinum ultramicroelectrodes. Anal. Chem. 2017, 89, 3087-3092.

[14] Noël, J.-M.; Yu, Y.; Mirkin, M. V. Dissolution of Pt at Moderately Negative Potentials during Oxygen Reduction in Water and Organic Media. Langmuir 2013, 29(5), 1346-1350.

[15] Bae, J. H.; Brocenschi, R. F.; Kisslinger, K.; Xin, H. L.; Mirkin, M. V. Dissolution of Pt during Oxygen Reduction Reaction Produces Pt Nanoparticles. Anal. Chem. 2017, 89(23), 12618-12621.

[16] Bard, A. J.; Fan, F. R. F.; Kwak, J.; Lev, O. Scanning electrochemical microscopy. Introduction and principles. Anal. Chem. 1989, 61, 132-138.

[17] Kwak, J.; Bard, A. J. Scanning electrochemical microscopy. Theory of the feedback mode. Anal. Chem. 1989, 61, 1221-1227.

[18] Polcari, D.; Dauphin-Ducharme, P.; Mauzeroll, J. Scanning electrochemical microscopy: a comprehensive review of experimental parameters from 1989 to 2015. Chem. Rev. 2016, 116, 13234-13278.

[19] Kwak, J.; Bard, A. J. Scanning electrochemical microscopy. Theory of the feedback mode. Anal. Chem. 1989, 61, 1221-1227.

[20] Ktari, N.; Poncet, P.; Senechal, H.; Malaquin, L.; Kanou, F.; Combellas, C. Patterning of polystyrene by scanning electrochemical microscopy. Biological applications to cell adhesion. Langmuir 2010, 26, 17348-17356. 
[21] Ktari, N.; Combellas, C.; Kanoufi, F. Local oxidation of polystyrene by scanning electrochemical microscopy. J. Phys. Chem. C 2011, 115, 17891-17897.

[22] Shiku, H.; Uchida, I.; Matsue, T. Microfabrication of alkylsilanized glass substrate by electrogenerated hydroxyl radical using scanning electrochemical microscopy. Langmuir 1997, $13,7239-7244$.

[23] Keene, J. P. Kinetics of Radiation-induced Chemical Reactions. Nature 1960, 188, 843844.

[24] Adams, G. E.; Boag, J. W. Spectroscopic Studies of Reactions of the OH radical. Proc. Chem. Soc. 1964, 112.

[25] Adams, G. E.; Boag, J. W.; Michael, B. D. Transient Spectra of Some Inorganic Radical Anions Produced by Reactions of the Hydroxyl Radical. Proc. Chem. Soc. 1964, 411.

[26] Evrard, D.; Lambert, F.; Policar, C.; Balland, V.; Limoges, B. Electrochemical functionalization of carbon surfaces by aromatic azide or alkyne molecules: a versatile platform for click chemistry. Chem. Eur. J. 2008, 14, 9286-9291.

[27] Combellas, C.; Jiang, D.-E.; Kanoufi, F.; Pinson, J.; Podvorica, F. I. Steric effects in the reaction of aryl radicals on surfaces. Langmuir 2009, 25, 286-293.

[28] del Campo, J. J.; Godignon, P.; Aldous, L.; Pausas, E.; Sarrion, M.; Zabala, M.; Prehn, R.; Compton, R. G. Fabrication of PPF electrodes by a rapid thermal process. J. Electrochem. Soc. 2011, 158, H63-H68. 
[29] Nunes Kirchner, C.; Hallmeier, K. H.; Szargan, R.; Raschke, T.; Radehaus, C.; Wittstock, G. Evaluation of thin film titanium nitride electrodes for electroanalytical applications. Electroanalysis 2007, 19, 1023-1031.

[30] Sklyan, O.; Wittstock, G.; Numes Kirchner, C.; Leash, A. SECMX Software; http://www.wittstock.chemie.uni-oldenburg.de/de/research/secm/SECMx.html

[31] Gwyddion Open Source Software: http://gwyddion.net/

[32] Belanger, D.; Pinson, J. Electrografting: a powerful method for surface modification. Chem. Soc. Rev. 2011, 40, 3995-4048.

[33] Chehimi, M. M. Aryl Diazonium Salts: New Coupling Agents in Polymer and Surface Science. John Wiley \& Sons: 2012.

[34] Garrat, P. J. Alkylations of alkynyl carbanions. In Comprehensive Organic Synthesis, pages 271-292. Pergamon, Oxford: 1991.

[35] Leroux, Y. R.; Fei, H.; Noël, J.-M.; Roux, C.; Hapiot, P. Efficient covalent modification of a carbon surface: use of a silyl protecting group to form an active monolayer. J. Am. Chem. Soc. 2010, 132, 14039-14041.

[36] Combellas, C.; Jiang, D.-E.; Pinson, J.; Podvorica, F. I. Steric effects in the reaction of aryl radicals on surfaces. Langmuir 2009, 25, 286-293.

[37] McCreery, R. L. Advanced carbon electrode materials for molecular electrochemistry. Chem. Rev. 2008, 108, 2646-2687. 
[38] DuVall, S. H.; McCreery, R. L. Control of catechol and hydroquinone electron transfer kinetics on native and modified glassy carbon electrodes. Anal. Chem. 1999, 71, 4594-4602.

[39] Ferrell, R. T.; Himmelblau, D. M. Diffusion coefficients of nitrogen and oxygen in water. J. Chem. Eng. Data 1967, 12, 111-115.

[40] Davis, R. E.; Horvath, G. L.; Tobias, C. W. The solubility and diffusion coefficient of oxygen in potassium hydroxide solutions. Electrochim. Acta 1967, 12, 287-297.

[41] Lingane, J. J. Chronopotentiometric study of oxygen reduction at a platinum wire cathode. J. Electroanal. Chem. 1961, 2, 296-309.

[42] Narita, E.; Lawson, F.; Han, K. N. Solubility of oxygen in aqueous electrolyte solutions. Hydrometallurgy 1983, 10, 21-37.

[43] Bard, A. J.; Mirkin, M. V. Scanning Electrochemical Microscopy; Marcel Dekker: New York, 2001.

[44] Anderson, R. F.; Schulte-Frohlinde, D. Reactions induced by hydroxyl radical attack on acetylene in aqueous solution. A pulse radiolysis study. J. Phys. Chem. 1978, 82, 22-26.

[45] Senosiain, J. P.; Klippenstein, S. J.; Miller, J. A. The reaction of acetylene with hydroxyl radicals. J. Phys. Chem. A 2005, 109, 6045-6055.

[46] Anbar, M.; Meyerstein, D.; Neta, P. Reactivity of aliphatic compounds towards hydroxyl radicals. J. Chem. Soc. B 1966, 742-747. 
[47] Dorfman, L. M.; Adams, G. E. Reactivity of the Hydroxyl Radical in Aqueous Solutions. National Bureau of Standards: 1973.

[48] Anderson, R. F.; Schulte-Frohlinde, D. Reactions induced by hydroxyl radical attack on acetylene in aqueous solution. A pulse radiolysis study. J. Phys. Chem. 1978, 22-26.

[49] Senosiain, J. P.; Klippenstein, S. J.; Miller, J. A. The reaction of acetylene with hydroxyl radicals. J. Phys. Chem. A 2005, 6045-6055.

[50] Anbar, M.; Meyerstein, D.; Neta, P. Reactivity of aliphatic compounds towards hydroxyl radicals. J. Chem. Soc. B 1966, 742-747.

[51] Kopinke, F.-D.; Georgi, A. What Controls Selectivity of Hydroxyl Radicals in Aqueous Solution? Indications for a Cage Effect. J. Phys. Chem. A 2017, 7947-7955.

[52] Zhang, C.; Fan, F.-R. F.; Bard, A. J. Electrochemistry of oxygen in concentrated NaOH solutions: solubility, diffusion coefficients, and superoxide formation. J. Am. Chem. Soc. 2008, 131(1), 177-181. 